\title{
Topoisomerase II alpha expression and the benefit of adjuvant chemotherapy for postoperative patients with non-small cell lung cancer
}

\author{
Shi Yan ${ }^{1}$, Jiao Shun-Chang ${ }^{1 *}$, Chen Li ${ }^{1}$, Li Jie ${ }^{2}$, Lv Ya-Li ${ }^{2}$, Wang Ling-Xiong ${ }^{3}$
}

\begin{abstract}
Background: Adjuvant chemotherapy has been shown to improve survival rates of postoperative patients with non-small cell lung cancer (NSCLC). Biomarkers could help select an appropriate chemotherapy for NSCLC patients or predict the efficacy of chemotherapy. The objective of this study was to explore the possible prognostic and predictive role of topoisomerase II alpha (Toplla) expression level in postoperative NSCLC patients who received adjuvant chemotherapy.
\end{abstract}

Methods: Patients with stage I-III NSCLC, who underwent surgery in our hospital from January 2004 to December 2007 and who also received adjuvant chemotherapy after surgery, were analyzed in this study. Expression of Toplla and Ki67 in paraffin-embedded tissues was detected by immunohistochemistry (IHC). The relationships between clinicopathological characteristics, chemotherapy regimens, the expression of biomarkers and disease free survival (DFS) were analyzed.

Results: Toplla and Ki67 were highly expressed in 22.5\% and 36.4\% of the 151 patients, respectively. Univariate survival analysis showed that male sex $(P=0.036)$, non-adenocarcinoma $(P=0.004)$, earlier pathological TNM stage $(P=0.001)$ or pathological $N$ stage $(P<0.001)$, and high expression of Toplla $(P=0.012)$ were correlated with better DFS, whereas age, smoking history, different chemotherapy regimens, T stage and expression level of Ki67 were of no prognostic significance. Further stratified analysis showed that vinorelbine (NVB)-containing adjuvant regimens were generally associated with better DFS than regimens without NVB in patients with low Toplla expression, though the difference was not statistically significant $(P=0.065)$. Pairwise comparisons for patients with low Topll $\alpha$ expression indicated that the NVB-containing regimen was associated with better DFS than the docetaxel (TXT)-containing regimen ( $P=0.047)$. COX multivariate analysis showed that pathological TNM stage, histological subtype and expression level of Topll $\alpha$ to be independent of risk factors affecting DFS in postoperative NSCLC patients who received chemotherapy.

Conclusions: High Toplla expression was discovered to be correlated with better DFS for postoperative NSCLC patients who received adjuvant chemotherapy. The NVB-containing chemotherapy regimen was more effective than the TXT-containing regimen in improving DFS in patients with low Toplla expression. Topllo could be considered to be an independent prognostic biomarker of DFS in postoperative NSCLC patients who received adjuvant chemotherapy.

\section{Background}

Non-small cell lung cancer (NSCLC) remains one of the most common cancers in China and in the world [1]. Although surgery is the main curative treatment,

\footnotetext{
* Correspondence: jiaosc@vip.sina.com

'Department of Medical Oncology, General Hospital of CPLA, No.28 FuXing Road, Beijing, China

Full list of author information is available at the end of the article
}

postoperative recurrence ranges from $30 \%$ to $50 \%$ [2]. Positive results of recent clinical trials, including the International Adjuvant Lung Cancer Trial (IALT), the National Cancer Institute of Canada Clinical Trials Group (NCIC CTG JBR.10) and the Adjuvant Navelbine International Trialist Association (ANITA), provide preliminary confirmation on the role of adjuvant chemotherapy, showing that platinum-based postoperative chemotherapy offers a
C Biomed Central

(c) 2010 Yan et al; licensee BioMed Central Ltd. This is an Open Access article distributed under the terms of the Creative Commons Attribution License (http://creativecommons.org/licenses/by/2.0), which permits unrestricted use, distribution, and reproduction in any medium, provided the original work is properly cited. 
significant 5-year survival benefit compared with surgery alone [3-5]. However, only $5 \%-15 \%$ of the individuals who received adjuvant chemotherapy achieved long-term survival improvement [6]. Therefore, it is very important to select subgroups of patients who are most likely to benefit from postoperative chemotherapy and who are potentially resistant to a given chemotherapy regimen. Recent studies have shown that expression of excision repair crosscomplementation group 1 (ERCC1), ribonucleotide reductase subunit M1 (RRM1) and class III beta-tubulin ( $\beta$-tubulinIII) could provide predictive value for patients treated with platinum agents, gemcitabine (GEMZ) and microtubule-interacting agents (including taxanes and vinorelbine (NVB)), respectively [7].

Topoisomerase II alpha (TopII $\alpha$ ) is a nuclear enzyme that catalyzes the conversion between DNA topological isomers and can be detected in cells with high proliferative activity. Many anticancer agents (i.e., anthracyclines) exert anticancer effects by stabilizing DNA cleavage and inhibiting DNA replication via binding and blocking the activity of TopII $\alpha$ [8]. TopII-inhibiting chemotherapeutic agents, including irinotecan, etoposide and topotecan, are commonly used for the treatment of small cell lung cancer (SCLC), but they are seldom used for the treatment of NSCLC. However, it has been reported that one type of multidrug resistance (MDR) in lung cancer, atypical MDR, is mediated through an altered expression of TopII. This type of resistance was found to be associated either with decreased TopII $\alpha$ expression or with a mutation that altered the interaction of the enzyme with the drug or DNA [9]. High TopII $\alpha$ expression has been observed in many kinds of cancers, including breast cancer and NSCLC [10-13]. Although TopII $\alpha$ expression and its implication for TopII inhibitors have been extensively investigated in several cancers, especially in breast cancer, few reports have described TopII $\alpha$ expression and its cross resistance to other cytotoxic drugs in NSCLC $[10,11,14-16]$. In addition, some studies have reported that low levels of TopII $\alpha$ gene or protein expression might be related with resistance not only to TopII inhibitors, such as etoposide and anthracycline, but also to other cytotoxic drugs, such as cisplatin, microtubule-interacting agents and gemcitabine, in some cancer cell lines or cancers [17-19]. However, little is known about the correlation between TopII $\alpha$ expression, which can mediate cross resistance, and the effect of common chemotherapy drugs, including cisplatin, microtubule-interacting agents and GEMZ, in postoperative NSCLC.

To assess whether TopII $\alpha$ might be a valuable biomarker in postoperative NSCLC patients who received chemotherapy, TopII $\alpha$ expression levels in tumor samples were evaluated, and the prognostic and therapeutic predictive roles of TopII $\alpha$ were analyzed. Ki67, another molecular marker that is related to proliferation, was evaluated as a control factor.

\section{Methods \\ Patient data}

Patients who underwent curative surgery for NSCLC between January 2004 and December 2007 received at least two cycles of adjuvant chemotherapy within two months after surgery in the General Hospital of Chinese People's Liberation Army (CPLA), and for whom a formalin-fixed, paraffin-embedded sample adequate for analysis was available, were included in the study. Patients who received neoadjuvant chemotherapy or neoadjuvant radiotherapy were excluded.

Data concerning clinicopathological features, including age, sex, smoking history, histological subtypes, pathological TNM stage, surgical methods, chemotherapy regimens and cycles, other treatments such as adjuvant radiotherapy, date of recurrence or metastasis, and survival information, were obtained by medical records, outpatient and telephone follow-ups. Outcome data including disease free survival (DFS) and overall survival (OS) were calculated. Clinical data were kept unavailable during immunohistochemistry (IHC). The study design and procedure involving the human tissue sample collection were reviewed and approved by the ethical board of the General Hospital of CPLA.

\section{Immunohistochemistry}

Representative tumor paraffin-embedded blocks were selected and collected, after two pathologists reviewed, the tumor sample slices stained with hematoxylin and eosin (HE). Sections ( $4 \mu \mathrm{m}$ thick) were cut from tumor specimens, placed onto 3-amino-propyltriethoxy-silane (APES) slides, and deparaffinized in xylene and gradient ethanol. Antigen retrieval was performed by placing slides in a high-pressure cooker in a $0.01 \mathrm{mM}$ citrate buffer, $\mathrm{pH}$ 6.0, for $2.5 \mathrm{~min}$ at $100^{\circ} \mathrm{C}$; they were then cooled for $20 \mathrm{~min}$. Endogenous peroxidase activity was blocked by incubating the section in $3 \% \mathrm{H}_{2} \mathrm{O}_{2}$ for $10 \mathrm{~min}$, followed by rinsing in PBS solution three times. Immunohistochemical staining was performed with the two-step EnVision(tm)+ System Kit (Dako, Denmark). The sections were incubated with mouse anti-TopII $\alpha$ monoclonal primary antibodies (clone 3F6, 1/75 dilution, Novocastra, UK) or mouse anti-Ki67 monoclonal primary antibodies (clone MIB1, 1/200 dilution, Dako) at $37^{\circ} \mathrm{C}$ for $60 \mathrm{~min}$, followed by dextran polymer conjugated with horseradish peroxidase enzyme and secondary anti-mouse antibody (Dako). Slides were stained with 3,3'-diaminobenzidine tetrahydrochloride (DAB) chromogen and counter-stained with hematoxylin. Negative controls were conducted by adding PBS solution instead of the primary antibody, and positive controls 
were conducted by staining known-positive samples from our pathology specimen bank.

All the specimens were examined and scored by two independent pathologists without the knowledge of patient data, and their inter-observer concordance was over $90 \%$. The number of positive cells in 1,000 tumor cells within 10 microscopic fields at $\times 200$ magnification was counted and scored as follows: 0 (negative, $<25 \%$ ), 1 (focal, $25 \%$ to $49 \%$ ), 2 (moderate, $50 \%$ to $74 \%$ ), 3 (diffuse, $\geq 75 \%$ ). Samples with scores of 0 and 1 were considered to have low expression, whereas samples of 2 and 3 were considered to have high expression.

\section{Statistical analysis}

Correlations between immunohistochemical expression and patient or tumor characteristics were performed by using the $\chi^{2}$ test or Fisher's exact test, as appropriate. Survival curves were estimated by the Kaplan-Meier method, and differences in DFS among groups were compared by using the log-rank test. The COX proportional hazards model was used for multivariate analysis to assess the independent value of TopII $\alpha$ expression. Two-sided P < 0.05 was considered statistically significant. All analyses were performed with the SPSS 16.0 software package.

\section{Results}

\section{Patient characteristics}

We enrolled 151 eligible patients (111 male and 40 female) in this study, who ranged in age from 31 to 81 years, with a median of 55 years, of whom $86(60 \%)$ had a history of smoking. The main histological types included squamous cell carcinoma (41.0\%), adenocarcinoma (39.1\%), bronchioloalveolar carcinoma (10.0\%), large cell carcinoma (6.6\%) and adenosquamous (3.3\%). Stage I, II and III were diagnosed in $35.8 \%, 31.1 \%$ and $33.1 \%$ patients, respectively. In addition, according to pathological $\mathrm{T}$ stage or $\mathrm{N}$ stage, T1, T2, T3 and T4 were diagnosed in $25.2 \%, 65.5 \% 3.3 \%$ and $6.0 \%$ patients, respectively, and N0, N1 and $\mathrm{N} 2$ were diagnosed in $41.1 \%, 33.1 \%$ and $25.8 \%$ patients, respectively. The majority had undergone lobectomy (81.5\%). All patients received 2 to 8 cycles of adjuvant chemotherapy, of whom 114 (75.5\%) finished at least 4 cycles. The main chemotherapy regimens included docetaxel (TXT, 26.5\%), GEMZ (33.8\%), NVB (26.5\%) and paclitaxel (PTX, 13.2\%) combined with or without cisplatin (DDP)/carboplatin (CBP). Of the 151 patients, 35 patients $(23.2 \%)$, most of whom were diagnosed with stage III or N2, received adjuvant radiotherapy. Detailed characteristics are shown in Table 1.

\section{Topll $\alpha$ and Ki67 expression in NSCLC}

TopIIa (Figure 1A and 1B) and Ki67 (Figure 1C and 1D) were located in the nucleus. TopII $\alpha$ and Ki67 were highly expressed in $22.5 \%$ and $36.4 \%$, respectively, of all 151 patients. Additionally, the expression of TopII $\alpha$ had a strongly positive correlation with that of Ki67 ( $\mathrm{r}=$ $0.515, \mathrm{P}<0.001$ ). Table 2 shows the correlation between TopII a and Ki67 expression levels and clinical characteristics, including sex, smoking history, histological subtypes, pathological TNM stage, pathological T stage and pathological N stage.

\section{Patient outcomes and clinical predictors}

After a median follow-up of 35.8 months (ranging from 16.4 - 63.7 months), 80 (53\%) patients had metastatic or recurrent tumors and $40(26.5 \%)$ patients had died. Causes of death were NSCLC (37 patients), radiation pneumonitis (1 patient), appearance of a secondary tumor (1 patient) and severe pulmonary infection (1 patient). The median DFS of all 151 patients was 24.3 months and the median OS was not reached. The primary purpose of the study was to assess the associations between clinical characteristics, TopII $\alpha$ protein expression and efficacy of adjuvant chemotherapy. DFS was more representative than OS as an indirect measure of efficacy, because OS was often affected by treatments other than adjuvant chemotherapy after recurrence or metastasis. DFS was therefore used as the main outcome measure in this study.

Univariate survival analysis showed that sex, pathological TNM stage, pathological N stage, histological subtype (adenocarcinoma or not) and TopII $\alpha$ expression level were prognostic factors related to DFS, while age, smoking history, surgical methods, pathological $\mathrm{T}$ stage, chemotherapy regimens and expression level of Ki67 were of no prognostic significance. Log-rank test indicated that male sex $(P=0.036$, Figure $2 A)$, earlier pathological TNM stage $(\mathrm{P}=0.001$, Figure $2 \mathrm{~B})$ and pathological $\mathrm{N}$ stage $(\mathrm{P}<0.001)$, non-adenocarcinoma $(\mathrm{P}=0.004$, Figure $2 \mathrm{C})$ and high TopII $\alpha$ expression $(\mathrm{P}=0.012$, Figure $2 \mathrm{D})$ predicted better DFS in postoperative NSCLC patients who received adjuvant chemotherapy. Although the $\mathrm{P}$ value for comparison of the survival curves in different $T$ stages was less than $0.05(P=0.03)$, there was no significant difference between $\mathrm{T} 1$ stage and T3-4 stage $(\mathrm{P}=$ $0.677)$ or between $T 2$ stage and T3-4 stage $(P=0.245)$ when pairwise comparisons of the three curves were performed. Only the difference between T1 stage and T2 stage was significant $(p=0.011)$. Detailed characteristics are also shown in Table 1.

\section{Correlations between Topll $\alpha$ expression and the value of adjuvant chemotherapy}

It was found that high expression of TopII $\alpha$ was significantly correlated with better DFS in the patients studied, suggesting that patients with high TopII $\alpha$ expression benefited more from the adjuvant chemotherapy than 
Table 1 Clinical and pathological characteristics and univariate analysis for DFS

\begin{tabular}{|c|c|c|c|c|}
\hline \multicolumn{2}{|c|}{ Factors } & \multirow{2}{*}{$\begin{array}{c}\text { Number (\%) } \\
111(73.5)\end{array}$} & \multirow{2}{*}{$\begin{array}{c}\text { Median DFS (months) } \\
33.99\end{array}$} & \multirow{2}{*}{$\frac{P \text { value }}{0.036^{*}}$} \\
\hline Sex & male & & & \\
\hline & female & $40(26.5)$ & 21.13 & \\
\hline \multirow[t]{2}{*}{ Age } & $<55$ years & $74(49.0)$ & 21.45 & 0.330 \\
\hline & $\geq 55$ years & $77(51.0)$ & 31.97 & \\
\hline \multirow[t]{2}{*}{ Smoking status } & no & $65(43.0)$ & 22.24 & 0.305 \\
\hline & yes & $86(57.0)$ & 32.99 & \\
\hline \multirow[t]{3}{*}{ Stage } & I & $54(35.8)$ & $N R^{\#}$ & $0.001^{*}$ \\
\hline & $\|$ & $47(31.1)$ & 33.64 & \\
\hline & III & $50(33.1)$ & 15.18 & \\
\hline \multirow[t]{3}{*}{ T stage } & $\mathrm{T} 1$ & $38(25.2)$ & $N R^{\#}$ & 0.03 \\
\hline & $\mathrm{T} 2$ & $99(65.5)$ & 18.76 & \\
\hline & T3-4 & $14(9.3)$ & 38.16 & \\
\hline \multirow[t]{3}{*}{ N stage } & No & $62(41.1)$ & $N R^{\#}$ & $<0.001^{*}$ \\
\hline & N1 & $50(33.1)$ & 33.64 & \\
\hline & $\mathrm{N} 2$ & $39(25.8)$ & 13.37 & \\
\hline \multirow[t]{2}{*}{ Histology } & Adenocarcinoma & $59(39.1)$ & 17.41 & $0.004^{*}$ \\
\hline & Non- adenocarcinoma & $92(60.9)$ & 48.16 & \\
\hline \multirow[t]{3}{*}{ Surgery method } & Wedge resection & $5(3.3)$ & 17.41 & 0.958 \\
\hline & Lobectomy & $123(81.5)$ & 24.41 & \\
\hline & Pneumonectomy & $23(15.2)$ & 31.15 & \\
\hline \multirow[t]{4}{*}{ Chemotherapy regimens } & Docetaxel-containing & $40(26.5)$ & 18.60 & 0.523 \\
\hline & Vinorelbine- containing & $40(26.5)$ & 48.16 & \\
\hline & Gemcitabine- containing & $51(33.8)$ & 24.58 & \\
\hline & Paclitaxel- containing & $20(13.2)$ & 24.41 & \\
\hline \multirow[t]{2}{*}{ Toplla } & Low expression & $117(77.5)$ & 19.38 & $0.012^{*}$ \\
\hline & High expression & $34(22.5)$ & NR & \\
\hline \multirow[t]{2}{*}{ Ki67 } & Low expression & $96(63.6)$ & 22.24 & 0.517 \\
\hline & High expression & $55(36.4)$ & 29.37 & \\
\hline
\end{tabular}

${ }^{*} \mathrm{P}<0.05$ was considered statistically significant; ${ }^{\text {N }} \mathrm{NR}=$ not reached.

those with low TopII $\alpha$ expression, and that the latter group of patients may be a potential chemotherapyresistant population. Further stratified analysis of the correlation between the value of adjuvant chemotherapy regimens and TopII $\alpha$ expression showed that when TopII $\alpha$ was highly expressed, there were no significant differences in DFS between patients who received chemotherapy with or without TXT $(\mathrm{P}=0.712)$, with or without NVB $(P=0.170)$, with or without GEMZ ( $P=$ $0.415)$, and with or without PTX $(P=0.763)$. In addition, when TopIIa expression was low, there were no significant differences in DFS between patients who received chemotherapy with or without TXT, with or without GEMZ, and with or without PTX (Table 3). However, in patients with low TopII $\alpha$ expression, the median DFS was 48.16 months for those who received NVB-containing chemotherapy regimens and 16.23 months for those who received regimens without $\mathrm{NVB}$ $(\mathrm{P}=0.065$, Figure $3 \mathrm{~A})$. Although this difference was marginally insignificant, it is possible that NVB- containing adjuvant chemotherapy is better than others in patients with low TopII $\alpha$ expression. To ascertain the predictive value of this observation with better certainty, the pooled comparisons as well as pairwise comparisons of DFS among four chemotherapy subgroups were investigated in patients with low and high TopIIa expression. In patients with high TopII $\alpha$ expression, no significant differences in DFS were observed among the four chemotherapy subgroups regardless of pooled or pairwise comparisons (Table 4). In patients with low TopII $\alpha$ expression, the $\mathrm{P}$ value was greater than 0.05 ( $\mathrm{P}$ $=0.267$, Figure $3 \mathrm{~B}$ ) for the pooled comparison of DFS among the four chemotherapy subgroups. Pairwise comparisons of the four survival curves showed no significant differences in $\mathrm{P}$ values between any two subgroups (Table 4 and Figure 3B), except the $P$ value between the NVB and TXT subgroups. Pairwise comparison for low TopII $\alpha$ expression indicated that the NVB-containing regimen was associated with better DFS than the TXTcontaining regimen $(\mathrm{P}=0.047)$. 


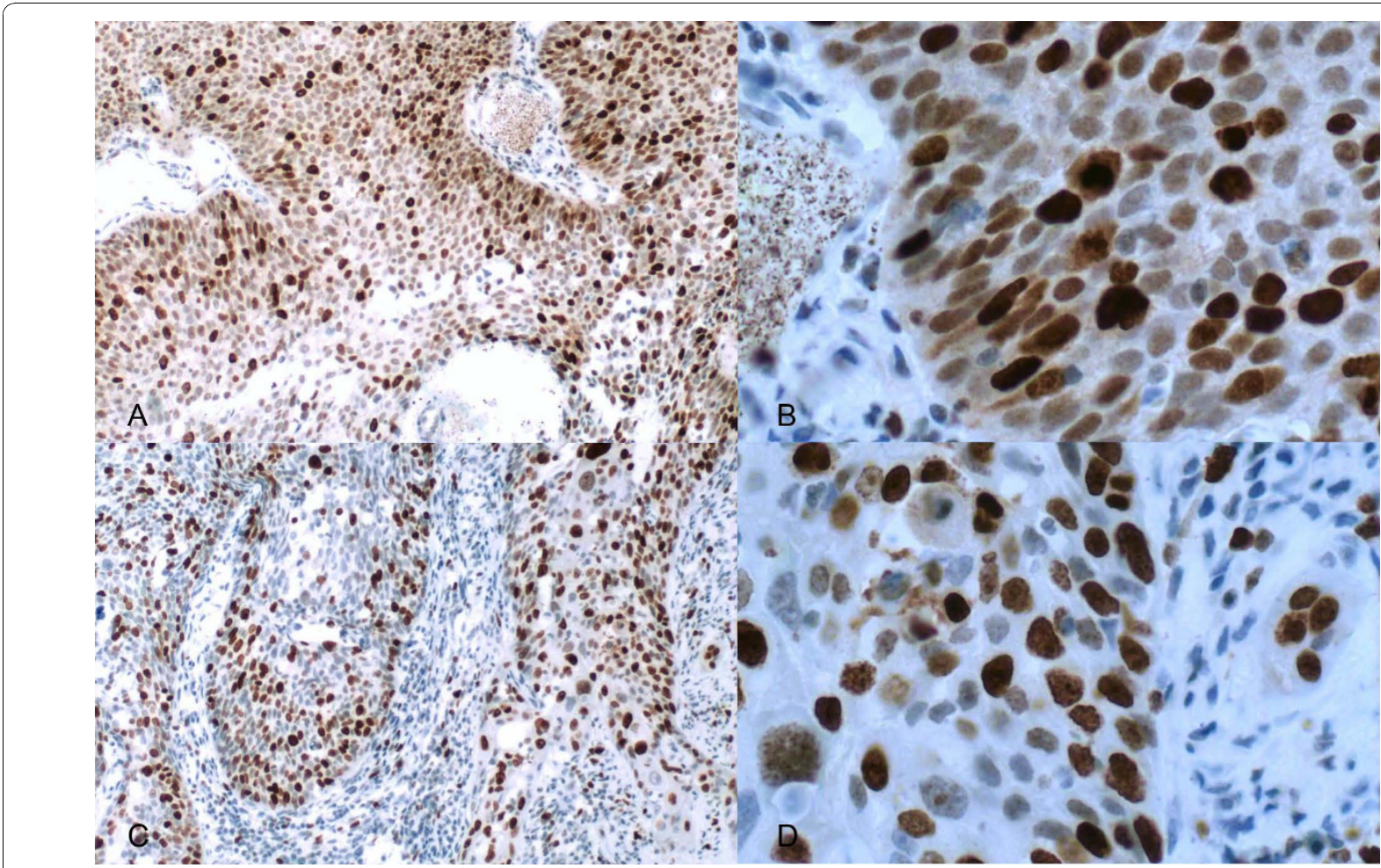

Figure 1 Positive expression of Topll $\alpha$ and Ki67 in non-small cell lung cancer by immunohistochemistry using the EnVision two-step method. (A) and (B) correspond to $\times 200$ and $\times 400$ magnification of Toplla respectively; (C) and (D) correspond to $\times 200$ and $\times 400$ magnification of Ki67 respectively.

\section{Multivariate analysis for DFS}

Multivariate analysis was performed with the COX proportional hazards model to explore whether the prognostic value of TopII $\alpha$ would disappear when other common prognostic factors were considered. Correlation analysis showed that pathological $\mathrm{T}$ stage and pathological TNM stage $(\mathrm{P}<0.001)$, and pathological $\mathrm{N}$ stage and pathological TNM stage $(\mathrm{P}<0.001)$ were mutually associated. As pathological TNM stage is more widely accepted as a DFS-related parameter than pathological $\mathrm{T}$ stage and $\mathrm{N}$ stage, subsequent multivariate analysis was performed to incorporate sex, histology, pathological TNM stage and TopII $\alpha$ expression level as covariates based on the significance of these factors in univariate analysis. The results from the Cox proportional hazard model using the forward stepwise method suggested that the histology subtype (adenocarcinoma vs. non-adenocarcinoma), TNM stage and the TopII $\alpha$ expression level were independent prognostic factors of DFS in NSCLC (Table 5). In postoperative patients who received adjuvant chemotherapy, high TopII $\alpha$ expression was a prognostic marker of longer DFS (hazard ratio 0.442 , 95\% confidence interval 0.239-0.818, $\mathrm{P}=0.009$ ), whereas adenocarcinoma (hazard ratio $2.140,95 \%$ confidence interval $1.373-3.337, \mathrm{P}=$ 0.001 ) and later TNM stage (hazard ratio $1.688,95 \%$ confidence interval 1.291-2.208, $\mathrm{P}<0.001$ ) were related to a shorter DFS as compared to non-adenocarcinoma and earlier TNM stage.

\section{Discussion}

The results of this study suggest that high TopII $\alpha$ expression in postoperative NSCLC patients who received adjuvant chemotherapy was associated with better DFS. However, other studies have reported that high TopII $\alpha$ expression was associated with a poorer survival rate in other types of tumors, including hypopharyngeal carcinoma, breast cancer and salivary gland carcinoma [20-23]. The reason for this difference is probably that all patients in this study received adjuvant chemotherapy while no patient received it in other studies. We therefore postulate that adjuvant chemotherapy might overcome the adverse biology of cancers that express higher TopII $\alpha$ protein. Additionally, other recent studies also showed that high expression or amplification of TopII $\alpha$ promised a good response to chemotherapy, particularly to topoisomerase inhibitors, which are partly consistent with our study $[20,24]$. The results of our study imply that patients with high TopII $\alpha$ expression might be able to obtain more benefits from adjuvant chemotherapy than those with low TopII $\alpha$ expression. Similar results were found in Sève et al.'s study of the prognostic role of $\beta$-tubulinIII in 
Table 2 Association between Topll $\alpha$ and Ki67 expression and clinical variables

\begin{tabular}{|c|c|c|c|c|c|c|}
\hline \multirow[t]{2}{*}{ Clinical variables } & \multicolumn{2}{|c|}{ Topll $\alpha$ expression (\%) } & \multirow[t]{2}{*}{$\mathbf{P}$} & \multicolumn{2}{|c|}{ Ki67 expression (\%) } & \multirow[t]{2}{*}{$\mathbf{P}$} \\
\hline & High & Low & & High & Low & \\
\hline \multicolumn{7}{|l|}{ Sex } \\
\hline Male & $25(22.5)$ & $86(77.5)$ & 0.998 & $39(35.1)$ & $72(64.9)$ & 0.584 \\
\hline Female & $9(22.5)$ & $31(77.5)$ & & $16(40.0)$ & $24(60.0)$ & \\
\hline \multicolumn{7}{|l|}{ Smoking status } \\
\hline No & $16(24.6)$ & $49(75.4)$ & 0.591 & $25(38.5)$ & $40(61.5)$ & 0.651 \\
\hline Yes & $18(20.9)$ & $68(79.1)$ & & $30(34.9)$ & $56(65.1)$ & \\
\hline \multicolumn{7}{|l|}{ Histology } \\
\hline Adenocarcinoma & $14(23.7)$ & $45(76.3)$ & 0.775 & $22(37.3)$ & $37(62.7)$ & 0.860 \\
\hline Non- adenocarcinoma & $20(21.7)$ & $72(78.3)$ & & $33(35.9)$ & $59(64.1)$ & \\
\hline \multicolumn{7}{|l|}{ Stage } \\
\hline । & $10(18.5)$ & $44(81.5)$ & 0.177 & $13(24.1)$ & $41(75.9)$ & $0.034^{*}$ \\
\hline$\|$ & 15 (31.9) & $32(68.1)$ & & $23(48.9)$ & $24(51.1)$ & \\
\hline III & $9(18.0)$ & $41(82.0)$ & & $19(38.0)$ & $31(62.0)$ & \\
\hline \multicolumn{7}{|l|}{ T stage } \\
\hline $\mathrm{T} 1$ & $13(34.2)$ & $25(65.8)$ & 0.148 & $18(47.4)$ & $20(52.6)$ & 0.168 \\
\hline $\mathrm{T} 2$ & $18(18.2)$ & $81(81.8)$ & & $34(34.3)$ & $65(65.7)$ & \\
\hline T3-4 & $3(21.4)$ & 11 (78.6) & & $3(21.4)$ & 11 (78.6) & \\
\hline \multicolumn{7}{|l|}{ N stage } \\
\hline NO & $12(19.4)$ & $50(80.6)$ & 0.137 & $16(25.8)$ & $46(74.2)$ & 0.065 \\
\hline N1 & 16 (32.0) & $34(68.0)$ & & $23(46.0)$ & $27(54.0)$ & \\
\hline N2 & $6(15.4)$ & $33(84.6)$ & & $16(41.0)$ & $23(59.0)$ & \\
\hline
\end{tabular}

${ }^{*} \mathrm{P}<0.05$ was considered statistically significant.
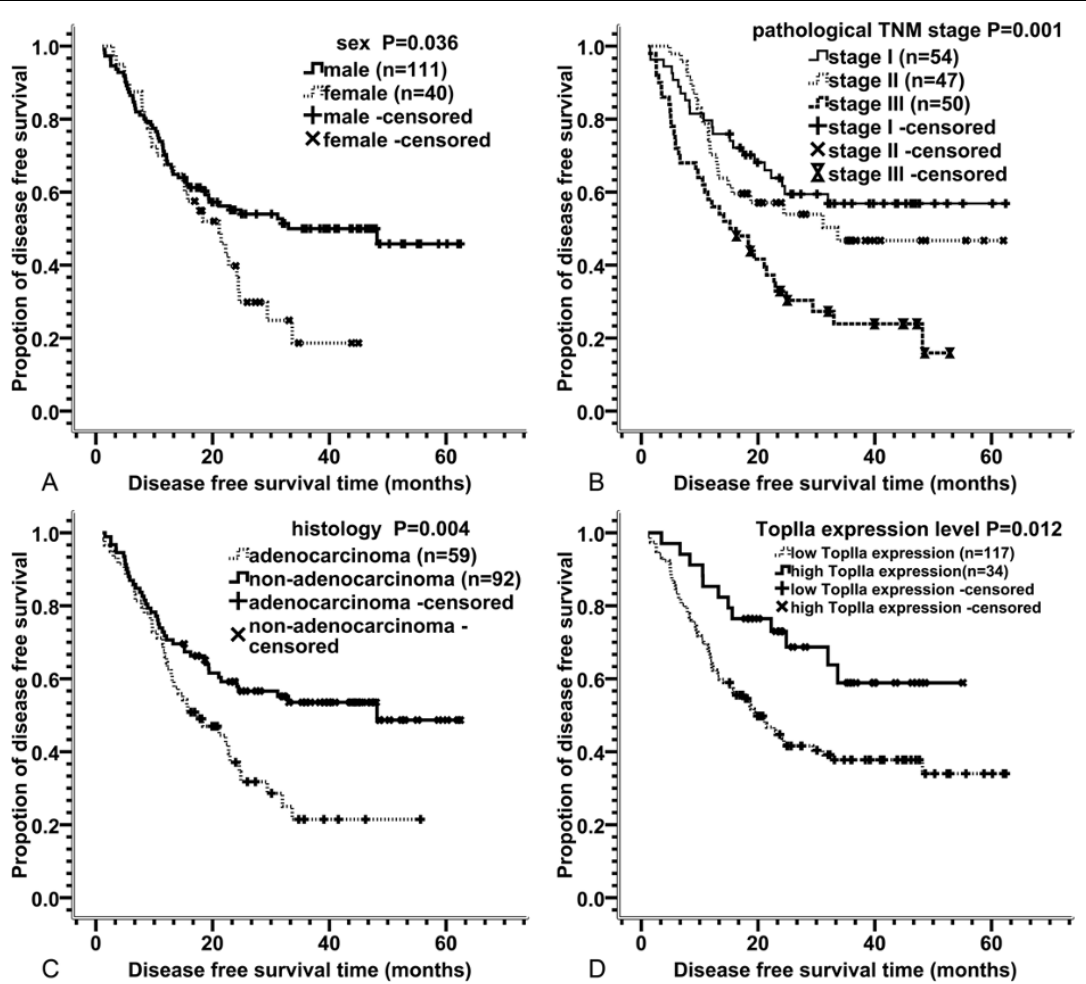

Figure 2 DFS curves according to different clinical and pathological characteristics. DFS curves according to: (A) sex; (B) pathological TNM stage; (C) histological subtype; (D) Toplla expression level. 
Table 3 Comparison of DFS among different chemotherapy groups in patients with low Topll $\alpha$ expression

\begin{tabular}{ccccc}
\hline Chemotherapy regimens & Number & Median DFS (months) & P \\
\hline Vinorelbine & Containing & 29 & 48.16 & 0.065 \\
& Not containing & 88 & 16.23 & \\
Gemcitabine & Containing & 39 & 14.13 & 0.530 \\
& Not containing & 78 & 22.74 & \\
Docetaxel & Containing & 33 & 15.74 & 0.194 \\
& Not containing & 84 & 22.74 & \\
Paclitaxel & Containing & 16 & 22.97 & 0.990 \\
& Not containing & 101 & 18.76 & \\
\hline
\end{tabular}

postoperative NSCLC [25]. Sève et al. discovered that high expression of $\beta$-tubulinIII was related to poor survival in the absence of adjuvant chemotherapy, though adjuvant chemotherapy prolonged the relapse free survival (RFS) and OS in patients with high $\beta$-tubulinIII expression. Therefore, $\beta$-tubulinIII acted as not only a prognostic biomarker of DFS but also a predictor of response to adjuvant chemotherapy. To identify predictive factors for survival benefit from treatment, an untreated control group should be included in the study, because a good survival identified in a single-arm trial might simply result from prognostic factors within the cohort, and might not be a result of treatment [26]. However, the major limitation of this study was that patients who did not receive adjuvant chemotherapy were not included. Therefore, we can merely draw the conclusion that TopII $\alpha$ acted as a prognostic biomarker of DFS in the postoperative NSCLC patients who received adjuvant chemotherapy. A further study including patients with and without adjuvant chemotherapy is required to clarify the predictive role of TopII $\alpha$ with respect to the benefit of adjuvant chemotherapy.
Moreover, TopIl $\alpha$ exhibited an independent significantly prognostic role for DFS, when common prognostic factors, including pathological TNM stage and histological subtype, were considered in multivariate analysis. In addition, the positive correlation between the expression of TopII $\alpha$ and that of Ki67, as observed in this study, is consistent with prior published reports in NSCLC and breast cancer $[13,27,28]$. It suggests that higher TopII $\alpha$ expression is associated with higher cell proliferation in NSCLC, as is the case with Ki67, which is a known cell proliferation marker. Although expression of Ki67 had a strongly positive correlation with expression of TopII $\alpha$, Ki67 was of no prognostic significance to DFS.

This is the first study reporting the evaluation of different adjuvant chemotherapy regimens by TopII $\alpha$ expression level in NSCLC. We found that NVB-containing regimens were associated with better DFS than those without NVB in patients with low TopII $\alpha$ expression, although the $\mathrm{P}$ value was marginally insignificant $(\mathrm{P}=$ 0.065). These results are concordant with previous reports related to the mechanism of atypical MDR, which is mediated by TopII $\alpha$ showing that atypical MDR exhibited cross resistance to many anticancer drugs, except vinca alkaloids $[29,30]$. However, the result of pooled and pairwise comparisons of DFS between the four chemotherapy subgroups for low or high TopII $\alpha$ expression showed that only the difference between the NVB and TXT subgroups was significant in patients with low TopII $\alpha$ expression $(\mathrm{P}=0.047)$. Therefore, it can be deduced that patients with low TopII $\alpha$ expression are a possible MDR population and that NVB-containing regimens may be better than TXT-containing regimens in these patients. Although there is no sufficient evidence that the efficacy of NVB is better than that of GEMZ and PTX in patients with low TopII $\alpha$ expression, DFS of the NVB subgroup was no worse than that of the GEMZ and
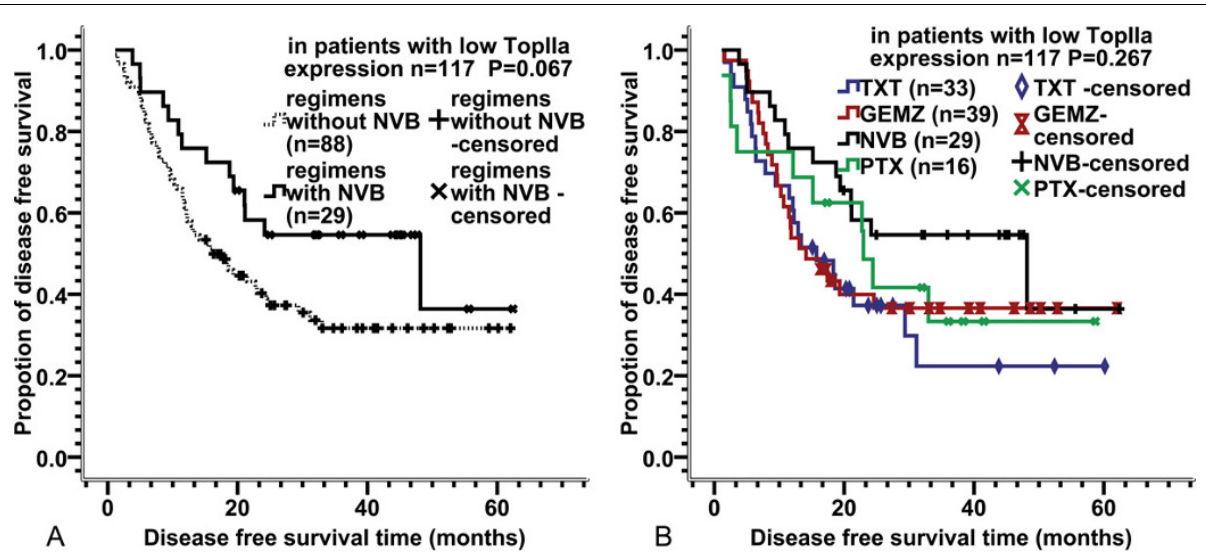

Figure 3 Comparison of DFS rates among different chemotherapy subgroups in patients with low Topll $\alpha$ expression. (A) depicts comparison of DFS curves between the NVB regimen group and the group of other regimens without NVB; (B) depicts comparison of DFS curves among four chemotherapy subgroups. 
Table 4 Pooled and pairwise comparisons of DFS among four chemotherapy regimens in patients with different Topll $\alpha$ expression levels

\begin{tabular}{|c|c|c|c|c|c|c|}
\hline Topll $\alpha$ expression & Chemotherapy regimens & Vinorelbine $(\mathrm{P})$ & Gemcitabine $(P)$ & Docetaxel (P) & Paclitaxel (P) & $P$ for each stratum \\
\hline \multirow[t]{4}{*}{ Low expression stratum } & Vinorelbine & & 0.128 & $0.047^{*}$ & 0.322 & 0.267 \\
\hline & Gemcitabine & 0.128 & & 0.676 & 0.786 & \\
\hline & Docetaxel & $0.047^{*}$ & 0.676 & & 0.429 & \\
\hline & Paclitaxel & 0.322 & 0.786 & 0.429 & & \\
\hline \multirow[t]{4}{*}{ High expression stratum } & Vinorelbine & & 0.209 & 0.410 & 0.437 & 0.594 \\
\hline & Gemcitabine & 0.209 & & 0.895 & 0.902 & \\
\hline & Docetaxel & 0.410 & 0.895 & & 0.987 & \\
\hline & Paclitaxel & 0.437 & 0.902 & 0.987 & & \\
\hline
\end{tabular}

${ }^{*} \mathrm{P}<0.05$ was considered statistically significant.

PTX subgroups (Figure 3B). In addition, in patients with high TopII $\alpha$ expression, there were no significant differences in DFS between the different regimens no matter what grouping or comparison methods were performed. Several studies have indicated that high TopII $\alpha$ expression was a positive predictive factor for response to anthracycline-based chemotherapy in patients with primary breast cancer [31,32]. It is well known that anthracycline in an anti-topoisomerase drug, and should exert strong anti-cancer activity in patients with high TopII $\alpha$ expression. However, further study is needed to determine whether an anti-topoisomerase-based adjuvant chemotherapy such as etoposide can really bring more benefits to postoperative NSCLC patients with high TopII $\alpha$ expression than other chemotherapy regimens.

Owing to the limitations of retrospective research, the conclusions drawn from this study need to be further identified and validated in prospective clinical trials. Another deficiency of this study is that the median OS was not reached due to limited follow-up time. Considering that the connection between TopIIa expression and adjuvant chemotherapy was the main purpose of this study, DFS was more representative than OS for evaluating efficacy. Moreover, OS could be influenced by many factors in this retrospective study, which may possibly lead to a negative result for long-term survival. Prospective randomized clinical

Table 5 Multivariate analysis for factors related to DFS using the COX proportional hazard model

\begin{tabular}{lcccc}
\hline \multicolumn{1}{c}{ Factors } & Wald & $\begin{array}{c}\text { Hazard } \\
\text { ratio }\end{array}$ & $\mathbf{9 5 \%} \mathbf{C l}$ & $\mathbf{P}$ \\
\hline Sex & 0.903 & 0.784 & $0.474-1.295$ & 0.342 \\
$\begin{array}{l}\text { Histology } \\
\text { (adenocarcinoma) }\end{array}$ & 11.277 & 2.140 & $1.373-3.337$ & $0.001^{*}$ \\
$\begin{array}{l}\text { TNM stage (later) } \\
\text { Topoisomerase II alpha }\end{array}$ & 14.631 & 1.688 & $1.291-2.208$ & $<0.001^{*}$ \\
$\begin{array}{l}\text { expression } \\
\text { (high expression) }\end{array}$ & & 0.442 & $0.239-0.818$ & $0.009^{*}$ \\
\hline
\end{tabular}

${ }^{*} \mathrm{P}<0.05$ was considered statistically significant. trials with larger sampling capacities and longer follow-up periods are needed to shed more light on the real relationship between TopII $\alpha$ expression and the benefit from the given chemotherapy.

This study demonstrated a significant association between TopII $\alpha$ expression and DFS, in which high TopII $\alpha$ expression was associated with better DFS than low TopII $\alpha$ expression, and NVB-containing adjuvant regimens were probably better than TXT-containing regimens in the low TopII $\alpha$ expressors. Thus, TopII $\alpha$ may be a good biomarker to predict the value of adjuvant chemotherapy, although further validation is required.

\section{Conclusions}

In conclusion, TopII $\alpha$ expression is an independent prognostic factor of DFS in postoperative NSCLC patients who received adjuvant chemotherapy. Low expression of TopIl $\alpha$ is related to poor DFS. NVB-containing adjuvant regimens are probably better than TXT-containing regimens in patients with low TopIIa expression. However, further investigation is needed to confirm the significance of TopII $\alpha$ as a prognostic or predictive biomarker before these conclusions can be translated into clinical practice.

\section{Acknowledgements}

This work was supported by the Key Project of the "Eleventh Five-year Plan" for Medical Science Development of PLA of China (Jiao Shun Chang, NO.06G106).

\section{Author details}

'Department of Medical Oncology, General Hospital of CPLA, No.28 FuXing Road, Beijing, China. 'Department of Pathology, General Hospital of CPLA, No.28 FuXing Road, Beijing, China. ${ }^{3}$ Cancer Center Laboratory, General Hospital of CPLA, No.28 FuXing Road, Beijing, China.

\section{Authors' contributions}

SY collected the clinical data, performed immunohistochemical staining and statistical analyses, and drafted the manuscript. CL and WLX participated in the coordination of the study. $L$ and $L Y L$ performed histological evaluation of immunohistochemical staining. JSC contributed to the conception and design of the study, interpretation of the data and revision of the manuscript. All authors have read and approved the final manuscript. 


\section{Competing interests}

The authors declare that they have no competing interests.

Received: 8 April 2010 Accepted: 10 November 2010

Published: 10 November 2010

\section{References}

1. Jemal A, Siegel R, Ward E, Hao Y, Xu J, Murray T, Thun MJ: Cancer statistics, 2008. CA Cancer J Clin 2008, 58:71-96.

2. Pisters KM, Le Chevalier T: Adjuvant chemotherapy in completely resected non-small-cell lung cancer. J Clin Oncol 2005, 23:3270-3278.

3. Arriagada R, Bergman B, Dunant A, Le Chevalier T, Pignon JP, Vansteenkiste J, International Adjuvant Lung Cancer Trial Collaborative Group: Cisplatin-based adjuvant chemotherapy in patients with completely resected non-small-cell lung cancer. N Engl J Med 2004, 350:351-360

4. Winton T, Livingston R, Johnson D, Rigas J, Johnston M, Butts C, Cormier Y, Goss G, Inculet R, Vallieres E, Fry W, Bethune D, Ayoub J, Ding K, Seymour L, Graham B, Tsao MS, Gandara D, Kesler K, Demmy T, Shepherd F, National Cancer Institute of Canada Clinical Trials Group; National Cancer Institute of the United States Intergroup JBR.10 Trial Investigators: Vinorelbine plus cisplatin vs. observation in resected non-small-cell lung cancer. $N$ Engl J Med 2005, 352:2589-2597.

5. Douillard JY, Rosell R, De Lena M, Carpagnano F, Ramlau R, GonzálesLarriba JL, Grodzki T, Pereira JR, Le Groumellec A, Lorusso V, Clary C, Torres AJ, Dahabreh J, Souquet PJ, Astudillo J, Fournel P, Artal-Cortes A, Jassem J, Koubkova L, His P, Riggi M, Hurteloup P: Adjuvant vinorelbine plus cisplatin versus observation in patients with completely resected stage IB-IIIA non-small-cell lung cancer (Adjuvant Navelbine International Trialist Association [ANITA]): a randomised controlled trial. Lancet Oncol 2006, 7:719-727.

6. Hotta K, Matsuo K, Ueoka H, Kiura K, Tabata M, Tanimoto M: Role of adjuvant chemotherapy in patients with resected non-small-cell lung cancer: reappraisal with a meta-analysis of randomized controlled trials. J Clin Oncol 2004, 22:3860-3867.

7. Singh N, Bal A, Aggarwal AN, Das A, Behera D: Clinical outcomes in nonsmall-cell lung cancer in relation to expression of predictive and prognostic biomarkers. Future Oncol 2010, 6:741-767.

8. Kellner U, Sehested M, Jensen PB, Gieseler F, Rudolph P: Culprit and victim -DNA topoisomerase II. Lancet Oncol 2002, 3:235-243.

9. Scagliotti GV, Novello S, Selvaggi G: Multidrug resistance in non-small-cell lung cancer. Ann Oncol 1999, 10(Suppl 5):S83-86.

10. Coon JS, Marcus E, Gupta-Burt S, Seelig S, Jacobson K, Chen S, Renta V, Fronda G, Preisler HD: Amplification and overexpression of topoisomerase lla predict response to anthracycline-based therapy in locally advanced breast cancer. Clin Cancer Res 2002, 8:1061-1067.

11. Kasahara K, Fujiwara Y, Sugimoto Y, Nishio K, Tamura T, Matsuda T, Saijo N: Determinants of response to the DNA topoisomerase II inhibitors doxorubicin and etoposide in human lung cancer cell lines. $J$ Natl Cancer Inst 1992, 84:113-118.

12. Dingemans AC, van Ark-Otte J, Span S, Scagliotti GV, van der Valk P Postmus PE, Giaccone G: Topoisomerase llalpha and other drug resistance markers in advanced non-small cell lung cancer. Lung Cancer 2001, 32:117-128.

13. Giaccone G, van Ark-Otte J, Scagliotti G, Capranico G, van der Valk P, Rubio G, Dalesio O, Lopez R, Zunino F, Walboomers J, Pinedo HM: Differential expression of DNA topoisomerases in non-small cell lung cancer and normal lung. Biochim Biophys Acta 1995, 1264:337-346.

14. Withoff $S$, de Vries EG, Keith WN, Nienhuis EF, van der Graaf WT, Uges DR, Mulder NH: Differential expression of DNA topoisomerase II alpha and -beta in P-gp and MRP-negative VM26, mAMSA and mitoxantroneresistant sublines of the human SCLC cell line GLC4. Br J Cancer 1996 74:1869-1876.

15. Koshiyama M, Fujii H, Kinezaki M, Yoshida M: Correlation between Topo II alpha expression and chemosensitivity testing for Topo II-targeting drugs in gynaecological carcinomas. Anticancer Res 2001, 21:905-910.

16. Cardoso F, Durbecq V, Larsimont D, Paesmans M, Leroy JY, Rouas G, Sotiriou C, Renard N, Richard N, Richard V, Piccart MJ, Di Leo A: Correlation between complete response to anthracyclinebased chemotherapy and topoisomerase II-a gene amplification and protein overexpression in locally advanced/metastatic breast cancer. Int J Oncol 2004, 24:201-209.
17. Giaccone G, Gazdar AF, Beck H, Zunino F, Capranico G: Multidrug sensitivity phenotype of human lung cancer cells associated with topoisomerase II expression. Cancer Res 1992, 52:1666-1674.

18. Skladanowski A, Côme MG, Sabisz M, Escargueil AE, Larsen AK: Downregulation of DNA topoisomerase llalpha leads to prolonged cell cycle transit in G2 and early M phases and increased survival to microtubuleinteracting agents. Mol Pharmacol 2005, 68:625-634.

19. Bai J, Sata N, Nagai H: Gene expression analysis for predicting gemcitabine sensitivity in pancreatic cancer patients. HPB (Oxford) 2007, 9:150-155

20. Kuropkat C, Rudolph P, Parwaresch R, Werner JA: Ki-S1-a prognostic marker for hypopharyngeal carcinoma with potential predictive value for response to chemotherapy. Anticancer Res 2003, 23:3965-3970.

21. Murphy AJ, Hughes CA, Barrett C, Magee H, Loftus B, O'Leary JJ, Sheils O: Low-level TOP2A amplification in prostate cancer is associated with HER2 duplication, androgen resistance, and decreased survival. Cancer Res 2007, 67:2893-2898.

22. Fritz P, Cabrera CM, Dippon J, Gerteis A, Simon W, Aulitzky WE, van der Kuip H: c-erbB2 and topoisomerase lla protein expression independently predict poor survival in primary human breast cancer: a retrospective study. Breast Cancer Res 2005, 7:R374-384.

23. Maruya S, Shirasaki T, Nagaki T, Kakehata S, Kurotaki H, Mizukami H, Shinkawa H: Differential expression of topoisomerase llalpha protein in salivary gland carcinomas: histogenetic and prognostic implications. BMC Cancer 2009, 9:72.

24. Pritchard Kl, Messersmith H, Elavathil L, Trudeau M, O'Malley F, DhesyThind B: HER-2 and topoisomerase II as predictors of response to chemotherapy. J Clin Oncol 2008, 26:736-744.

25. Sève $P$, Lai R, Ding K, Winton T, Butts C, Mackey J, Dumontet C, Dabbagh L, Aviel-Ronen S, Seymour L, Whitehead M, Tsao MS, Shepherd FA, Reiman T: Class III beta-tubulin expression and benefit from adjuvant cisplatin/ vinorelbine chemotherapy in operable non-small cell lung cancer: analysis of NCIC JBR.10. Clin Cancer Res 2007, 13:994-999.

26. Coate LE, John T, Tsao MS, Shepherd FA: Molecular predictive and prognostic markers in non-small-cell lung cancer. Lancet Oncol 2009, 10:1001-1010.

27. Nakopoulou L, Lazaris AC, Kavantzas N, Alexandrou P, Athanassiadou P, Keramopoulos A, Davaris P: DNA topoisomerase II-alpha immunoreactivity as a marker of tumor aggressiveness in invasive breast cancer. Pathobiology 2000, 68:137-143.

28. Rudolph P, MacGrogan G, Bonichon F, Frahm SO, de Mascarel I, Trojani M, Durand M, Avril A, Coindre JM, Parwaresch R: Prognostic significance of Ki67 and topoisomerase llalpha expression in infiltrating ductal carcinoma of the breast. A multivariate analysis of 863 cases. Breast Cancer Res Treat 1999, 55:61-71.

29. Beck WT, Cirtain MC, Danks MK, Felsted RL, Safa AR, Wolverton JS, Suttle DP, Trent JM: Pharmacological, molecular, and cytogenetic analysis of "atypical" multidrug-resistant human leukemic cells. Cancer Res 1987, 47:5455-5460.

30. Patel S, Fisher LM: Novel selection and genetic characterisation of an etoposide-resistant human leukaemic CCRF-CEM cell line. Br J Cancer 1993, 67:456-463.

31. Schindlbeck C, Mayr D, Olivier C, Rack B, Engelstaedter V, Jueckstock J, Jenderek C, Andergassen U, Jeschke U, Friese K: Topoisomerase II alpha expression rather than gene amplification predicts responsiveness of adjuvant anthracycline-based chemotherapy in women with primary breast cancer. J Cancer Res Clin Oncol 2010, 136:1029-1037.

32. Glynn RW, Miller N, Whelan MC, Kerin MJ: Topoisomerase 2 Alpha and the Case for Individualized Breast Cancer Therapy. Ann Surg Oncol 2010, 17:1392-1397.

\section{Pre-publication history}

The pre-publication history for this paper can be accessed here: http://www.biomedcentral.com/1471-2407/10/621/prepub

doi:10.1186/1471-2407-10-621

Cite this article as: Yan et al:: Topoisomerase II alpha expression and the benefit of adjuvant chemotherapy for postoperative patients with nonsmall cell lung cancer. BMC Cancer 2010 10:621. 